\title{
Milsark's Generalization and Categorical Judgments
}

\author{
S.-Y. Kuroda \\ University of California, San Diego
}

\section{The objective of the paper}

Milsark's generalization is well known.

Milsark's generalization "Properties may only be predicated of 'strong' NPs." (Milsark 1977:16).

In this paper, I shall restrict myself mostly to the discussion of bare NPs. I shall reformulate Milsark's generalization with this restriction and in terms of the individual-level (IL) and stage-level (SL) distinction introduced by Carlson (1977). But I expect that the main claims to be made below can be extended to indefinite NPs in general.1

Milsark's generalization (restricted to bare NPs and rephrased in terms of IL) "IL predicates may only be predicated of generic NPs."

Counterpart. "SL predicates may be predicated of existential NPs."

Milsark's generalization has generally been assumed to be related to the distinction between categorical and thetic judgments. The following generalization in the theory of judgments must be responsible for this perception:

The definiteness effect on categorical judgments 2

(a) The subject of a CATEGORICAL JUDGMENT must be a "semantically definite" (including "generic") noun phrase.

(b) The subject of a CATEGORICAL JUDGMENT may not be an indefinite "existential/cardinal" noun phrase.

The main objective of this paper is to challenge the validity of Milsark's generalization. According to the currently prevailing view, sentences with bare NP subjects represent different types of propositions depending on whether they are SL or IL; they are mapped onto different types of semantic representations. In the former, subject NPs are existentially quantified and in the latter they are bound by the generic operator. I will present evidence against this view and argue that the distinction between SL and IL cuts across the distinction between generic and existential construals of bare NP subjects. I will maintain that sentences with bare NP subjects, whether SL or IL, are subject to generic/universal or nongeneric/existential construals depending on the domain of interpretation.

\section{Preliminaries}

It is well known that there are two sentence types in Japanese that correspond to English declarative sentences: I call one the PLAIN SENTENCE and the other the $w a$ MARKED SENTENCE. Examples of plain sentences are given in (1)-(2) and examples of $w a$-marked ones are given in (3) - (5). The $w a$-marked noun phrase is commonly called a topic. I will follow this terminology, but I do not believe that the particle wa 
of the wa-marked sentence can be accounted for as a topic marker in the sense understood in discourse theory or information packaging. But I am not going to be concerned with that issue in this paper. See Kuroda (1992: 15f, 70ff) and Kuroda (2001).

(1) gengogaku no gakusei ga kinben de aru

linguistics GEN student NOM hard-working be

'linguistics students are hard-working'

inu ga neko o oikakete iru

dog NOM cat ACC chase be

'dogs are chasing cats'

(3) gengogaku no gakusei wa kinben de aru

linguistics GEN student TOP hard-working is

'linguistics students are hard-working'

inu wa neko o oikakete iru

dog cat ACC chase is

'dogs are chasing cats'

neko wa inu ga oikakete iru

cat dog NOM chase is

'cats, dogs are chasing'

In principle, any argument as well as any adjunct can be made a $w a$ topic. The syntactic subject is made a topic in (3)-(4), and the direct object in (5). For the sake of this paper I assume the syntactic derivation of $w a$-marked sentences from plain sentences by movement as given in (6). (3)-(5) are derived by this movement as suggested in (7)-(9).

$$
\begin{aligned}
& {[\mathrm{CP} . . .[\mathrm{VP} \ldots \mathrm{DP}} \\
& \mathrm{i} . . . . . . .] \\
& {[\text { gengogaku no gakusei wa }[\mathrm{t} \text { kinben de aru }]]} \\
& {[\text { inu wa }[\mathrm{t} \text { neko o oikakete iru] }]} \\
& {[\text { neko wa [inu ga t oikakete iru] }]}
\end{aligned}
$$

Semantically, the wa-marked sentence cannot be truth-conditionally distinguished from the plain sentence from which it is derived. To account for the functional difference between wa-marked and plain sentences, I have introduced and distinguished two semantic/cognitive objects, PROPOSITIONS and JUDGMENTS. I follow the common assumption that sentences/clauses represent PROPOSITIONS. In addition, I assume that sentences also express JUDGMENTS in syntactic contexts where they make assertions.

I assume that $w a$-marked and plain sentences express different types of judgments. A wa-marked sentence expresses a categorical judgment, that is, a judgment in the form of a predication. A plain sentence expresses a judgment of the nonpredicational form, a DESCRIPTION of an abstract or concrete situation; the THETIC JUDGMENT is by definition a subtype of description, a description of an event. In some embedded contexts, for example in conditional clauses, sentences/clauses do not make assertions; in such contexts only plain sentences can occur, and they are assumed to represent propositions but not to express any judgments. For this presentation, I have to assume that these distinctions are well known. 3

In what follows, however, most of the time we can assume the common semantic understanding according to which sentences are representations of propositions. The distinction between propositions and judgments matters only when I explicitly make reference to the concept of judgment. To sum up: 
(10)a Plain sentences may express descriptive/thetic judgments and represent propositions

b or simply represent propositions.

(11) Wa-marked sentences express categorical judgments and represent propositions.

\section{The interpretation of bare NP subjects of SL sentences}

3.1. Let us consider the following SL sentence:

(12)a ringo ga kago no naka ni kireini narabete atta apple NOM basket GEN in at beautifully arrange was

b 'apples were beautifully arranged in a basket'

The standard translation of (12) into formal logic is an existential proposition given in (13):

$\exists x[\operatorname{APPLE}(\mathrm{x}) \&$ ARRANGED-IN-A-BASKET(x)]

However, I would like to maintain that there are contexts in which (12) must be understood as if it represents a universally quantified proposition. Consider the following inference:

(14) (ano paathii de) ringo ga kago no naka ni kireini narabete atta; dakara (that party at) apples NOM basket GEN in at beautifully arrange was; therefore daremo ringo o tabe nakatta nobody apple ACC eat neg-past '(at that party) apples were beautifully arranged in a basket; therefore nobody ate apples'

Here, two sentences are connected by means of dakara 'therefore'; this conjoined sentence can give an impression that a natural inference was made. The background of the reasoning is the common-sense understanding that decent people refrain from disturbing what they perceive as a decorative arrangement. But there must be another "logical" basis underlying this natural reasoning: that the apples arranged in a basket are all the apples that there were. (13), though true, is not sufficient to describe the logical function of (12) in (14).

This point can be substantiated by observing the following subtle and interesting contrast. Let us first translate the existential formula (13), the standard logical rendition of (12), back into Japanese, literally by using the verb of existence atta 'was/were'. We get:

(15)a kago no naka ni kireini narabete aru ringo ga atta basket GEN in at beautifully arrange be apple NOM was

b 'apples that were beautifully arranged in a basket were/existed'

If we replace the first sentence (the premise) of the inference in (14) by (15), we get:

(16) (ano party de) kago no naka ni kirei ni narabete aru ringo ga atta; dakara daremo ringo o tabenakatta

'(at the party) apples that were beautifully arranged in a basket were/existed; therefore nobody ate apples' 
The inferences (14) and (16) should be logically equivalent; we have simply replaced one premise with another, which are supposedly logically equivalent. However, there is a subtle, but remarkable difference between (14) and (16). The same difference can be observed in English between (17)a and (17)b:

(17)a Apples were arranged beautifully in a basket; so, nobody ate apples.

b There were apples arranged beautifully in a basket; so nobody ate apples.

(16), and I believe (17)b as well, lack the degree of naturalness of inference we observe in (14) as well as (17)a. It is difficult, or impossible, to understand ringo 'apples' in (16) and (17)b as universally quantified. It is harder in (16) and (17)b than in (14) and (17)a to infer from the context that there were no apples other than those in the basket. Let us put aside for the moment why we get this difference, but the observed difference strengthens our initial intuition about (14); that is, ringo in (14) can be understood as universally quantified.

But it is definitely not the case that the subject ringo in (12) must be interpreted as universally quantified in all contexts. It is possible to witness an "inference" that contradicts such an interpretation.

(ano paathii de) ringo ga kago no naka ni kireini narabete atta; daremo (that party at) apples NOM basket GEN in at beautifully arrange was; nobody kago no naka no ringo o tabe nakatta

basket GEN in at apple ACC eat neg-past

'(at the party) apples were beautifully arranged in a basket; nobody ate apples arranged in the basket.'

By saying "nobody ate apples arranged in the basket" rather than "nobody ate apples," the speaker would naturally be taken as implying that people might have eaten apples but those were apples that were not arranged in the basket. To summarize, some uses of (12) require universal interpretation of ringo 'apples' but other uses contradict such an interpretation. Nonetheless, I would like to maintain that we have one and the same proposition represented by (12), and the difference in question is the matter of domains chosen for the interpretation of the proposition.

3.2. As a general description of the semantics of an SL sentence $S$ with a bare NP subject like (12), I propose the following formula:

$\mathrm{S}=\mathrm{NP}_{1} \ldots$ Pred...e...,

where $\mathrm{e}$ is an event argument and $\mathrm{NP}_{1}$, the subject of the predicate.

Interpretation:

$\left|\mathrm{NP}_{1}\right|_{\mathrm{S}, \mathrm{w}}=\left|\mathrm{NP}_{1}\right| \cap|\mathrm{e}|$ where $|\quad|$ indicates the "universal" valuation and ||$_{S, w}$, the interpretation relative to $S$ and a model/domain $w$ with respect to which the situation in question is described. $|\mathrm{e}|$ denotes the set of entities involved in e.

Let us agree that (19) presupposes that w contains e; only in such a domain is $S$ interpretable. A domain $w$ is called a g-DOMAIN for the proposition $\mathrm{p}$ represented by an SL sentence $S$ (or, simply, for $S$ ) if $\left|N P_{1}\right| S, w=\left|N P_{1}\right| \cap|w|$. Thus, a domain $w$ is a g-domain for $\mathrm{p}$, if and only if those "NP 1 's" that participate in e are exactly those that exist in $\mathrm{w}$. Note that whether a domain is a g-domain for $\mathrm{p}$ or not depends on the interpretation of the event argument $\mathrm{e}$. If a domain is not a g-domain, we call it a NON-g-DOMAIN. 4 
Let us consider sentence (12) in the context of the natural inference (14), and examine how it is understood. The hearer understands that an inference based on universal quantification is intended with respect to the subject, ringo 'apples'. Hence, the proposition $\mathrm{p}$ represented by (12) must be interpreted with respect to a gdomain. The domain may be the entire building where the party was held, or as small as the corner of the room where the speaker and hearer were located at the time of the utterance; in any event the domain is sufficiently small not to contain any apples other than those arranged in the basket in question. In contrast, (12), given in the context of discourse (18), must be understood as interpreted with respect to a non-g-domain of (12).

The current common assumption is that the semantic representation (the logical form) of an SL sentence with a bare NP subject is an existentially quantified form like (13). This assumption does not account for the understanding of (12) in (14). In order to account for this new situation, the common practice of formal semantics would require that sentence (12) be assigned another logical representation, i. e., a universally quantified form. One and the same sentence (12) would thus be assigned two logical forms; the sentence would be considered as semantically ambiguous. Instead of taking such a step, I propose that one and the same semantic interpretation corresponds to (12), as indicated by (19). Then, the difference in the logical function of (12) observed in (14) and (18) is a matter of different pragmatic choices of domains with respect to which the sentence is construed. If the domain is a g-domain, (12) can be an input to a universal type of inference. If not, it cannot be, and it is subject to the usual existential construal given by (13).

Semantic representations and model-theoretic interpretations are matters of grammar, but the choice of the domain $w$ with respect to which an interpretation is executed is a matter of the creative aspect of language use. Not only do choices constantly change during language use, but also they are ambiguous, not well demarcated and hard to digitize. Logical laws are valid only if $w$ is kept constant through a discourse. This constraint is, practically, hard to follow. In particular, gdomains for an event are generally very small, and it is difficult/rare for a discourse to proceed with such a small discourse domain being kept constant. Empirical evidence for g-domains may be hard to come by.

Whether w is a g-domain or not, if (12) is truthfully asserted, (13) holds, and vice versa. Thus, in this sense, (13) gives an adequate truth condition for (12). But if $w$ is a g-domain, (13) is an understatement and fails to monitor possible arguments done with (12) in natural language. Insomuch as the LOGICAL representation of a proposition represented by a sentence is assumed to model reasoning in natural language performance, we have to assume either that (12) is semantically ambiguous, representing two different propositions, or that we cannot assign a unique logical representation to THE proposition represented by (12). I am arguing for the second alternative and against taking (13) as THE semantic representation of (12). Put in general terms, my argument is that the logical representation of an SL sentence is a matter of pragmatics and does not belong to the level of semantic representation.

3.3. To recall, the contrast between the two inference forms, a natural one (14) and an inadequate one, (16), was crucial for our judgment that a bare NP subject may be construed as universally quantified. In order to account for this contrast, let us assume that the default choice for the domain of interpretation of an SL sentence with a bare NP subject is a g-domain.

This is a pragmatic default case assumption. Unless the pragmatic situation contradicts it, one interprets an SL sentence S by choosing a sufficiently small domain, so that it satisfies the condition for a g-domain. We might try to 
accommodate our utterance to meet this condition. Imagine, for example, that you are standing at a corner of a kitchen-dining area and see apples arranged in a basket on a table but we also see apples put on plates on the kitchen counter with other fruits. In this context, according to this assumption, (21) would be preferred to (20):

apples are arranged beautifully in a basket

on the table, apples are arranged beautifully in a basket

The locative explicitly suggests that the intended domain of interpretation is limited to a neighborhood of the table, excluding the counter area, a g-domain. 5

According to our assumption, the unmarked choice for the domain of interpretation for (12), the premise in (14), is a g-domain w of (12): There are no apples in w other than those arranged in a basket as described by (12). Given this understanding, (14) is a natural inference. Now, consider (15). The subject of (15) is kago no naka ni kirei ni narabete aru ringo, 'apples arranged beautifully in a basket', not ringo 'apples'. What is a g-domain of (15)? A domain W is a g-domain for (15) if and only if $\mathrm{W}$ does not contain any apples arranged beautifully in a basket other than those arranged in the basket referred to in (15); W may contain other apples not arranged in a basket. Hence, with (15) as a premise, a universal inference about apples arranged in a basket could be justified but not one about apples. As a consequence, (16) does not sound like a natural inference, as (14) does.

(12) and (15) stand in a relation worthy of particular note. They are truthconditionally equivalent. Interpreted with respect to the same situation/event e and with the same domain $\mathrm{D}$, they are either true or false together. Nonetheless, they are not logically equi-functional. A proper inference with (12) as a premise ceases to SOUND proper if the premise is replaced by the truth-conditionally equivalent (15). For, with (15), we are expected to interpret the inference with respect to a g-domain of (15). But, in fact, for (15), any domain is necessarily a g-domain, due to the special property of the predicate exist: no domain can contain apples that are beautifully arranged in a basket that do not exist. It follows that (15) is unprejudiced as to whether there are apples other than those that are arranged in a basket, and (16) cannot make a natural inference in any circumstances. We have thus accounted for the functional difference between the truth-conditionally equivalent (12) and (15).6

\subsection{The following assertions follow directly from (19):}

Assertion 1. (Downward invariance of the g-domain) A subdomain of a gdomain for an SL proposition $\mathrm{p}$ with a bare NP subject is a g-domain for $\mathrm{p} .{ }^{7}$

Assertion 2. (Upward invariance of the non-g-domain) A domain that contains a non-g-domain for an SL proposition $\mathrm{p}$ with a bare NP subject is a non-g-domain for $\mathrm{p}$.

Now I claim the following main assertion of this section to hold:

Assertion 3. An SL sentence S represents one and the same PROPOSITION through upward extension and downward restriction of domains.

Thus, with this assertion I contend that an SL sentence with a bare noun phrase subject has a unique semantic representation, even though the logical function of its subject is not invariant with changes of domains. One could take this assertion either as a proposal for a way to use the term "proposition" (a definition) or as a claim as to how to understand what "proposition" is (a hypothesis). The reason that I put it as an assertion (thus a hypothesis and an empirical claim) is that I assume we as 
theoreticians have some intuitive feel about "something" that we would wish to call a proposition and Assertion 3 is about this something. It claims that if the subject of an SL sentence is a bare noun phrase, its apparent function changes between gdomains and non-g-domains, but the sentence represents one and the same "proposition"; it is not SEMANTICALLY AMBIGUOUS. The speaker and hearer are to be warned that for a proper application of logic, they must keep track of the changes of domains of interpretation properly during a discourse, rather than of the changes of propositions represented by one and the same sentence.

So far the Japanese sentences we have dealt with are all plain sentences. Let us at this point consider wa-marked sentences. Consider (22):

(22) ringo wa kago no naka ni kireini narabete atta.

apple top basket gen in at beautifully arrange were 'apples were beautifully arranged in a basket'

Let us substitute (22) for (12) in (14):

(23)a (ano paathii de) ringo wa kago no naka ni kirei ni narabete atta; dakara daremo ringo o tabenakatta

b '(at that party) [the] apples were arranged beautifully in a basket; so, nobody ate apples'

I translated ringo wa as '[the] apples', with the definite article, but as there is no syntactic distinction between definite and indefinite in Japanese, it is a moot point to assume that the wa phrase in (23) here must be categorially taken as definite. 8 What interests us, and what is not moot, is that the wa-marking carries the implication that the apples in the basket were all the apples there were. Thus, (23) sounds as natural as, indeed perhaps more natural than, (14) as a deduction based on the common-sense presupposition that decent people do not disturb a decorative arrangement.

Compare (18) with (24) below. I replaced ga in (18) by wa.

(24) (ano paathii de) ringo wa kago no naka ni kirei ni narabete atta; daremo kago no naka no ringo o tabe nakatta

'(at that party) (the) apples were beautifully arranged in a basket; nobody ate apples in the basket.'

(18) invites the inference that there were other apples than those that were arranged in the basket. In contrast, (24) does not naturally invite such an inference. Rather, the $w a$ after the first ringo suggests that the apples in the basket were all the apples that there were. With this understanding, the inference would go through, though one might at least sense stylistic awkwardness: the direct object of the second sentence sounds redundantly modified by kago no naka no 'in the basket'.

These observations show that the wa-marked sentence (22) must be interpreted in a g-domain. I take (23) -(24) as evidence for the following assertion.

Assertion 4. A wa-marked SL sentence with a bare noun phrase subject is interpreted in a g-domain of the proposition represented by its plain sentence source. 


\section{The interpretation of bare NP subjects of IL sentences}

We now move to IL sentences. Consider the following discourse (25):

(25)a France-go wa iroirona kuni no hito ga hanasite iru France-language various country GEN person NOM speak are 'persons of various countries speak French'

b (Motiron,) France-zin ga France-go o hanasu of course France-people NOM France-language ACC speak '(Of course) The French speak French'

c (sorekara) Morocco-zin ga France-go o hanasu and Morocco-people NOM France-language ACC speak '(and) 'Moroccans speak French'

d Canada-zin ga France-go o hanasu. Canada-people NOM France-language ACC speak 'Canadians speak French'

The intended meaning of (25)d is not "generic/universal," but only "existential." The possibility of this non-generic/non-universal construal of a plain IL sentence contrasts sharply with the impossibility of such a construal for a wa-marked IL sentence. (28) is approximated only by (29); it's construal cannot be "nongeneric/non-universal" as (26) can. Thus, the plain sentence (26) can be used as an "existential" statement about Canadians in this real world, as in discourse (25), while (28) in contrast cannot be truthfully uttered about Canadians in general, given the way the real world is. (28) can of course be truthfully uttered, but only in special circumstances where all Canadians present happen generally to be French-speaking.

Canada-zin ga France-go o hanasu $\quad[=(25 d)]$

Keep in mind that the contrast between the wa-marked and the plain sentences is not between generic/universal and non-generic/non-universal interpretations; the plain sentence form, like (25)b-d, does not exclude the possibility of a generic/universal interpretation. Rather, the syntactic and pragmatic context determines the interpretation. Take, for example, the plain sentence form (25)b. Even in the context of discourse (25), this sentence can be taken as intended to be a generic/universal statement; it could in fact be continued as follows:

France-zin ga France-go o hanasu; zissai, France-zin wa France -people NOM France-language ACC speak; in fact, France-people France-go sika hanasanai France-language except speak-neg 'The French speak French; in fact, the French speak only French.'

Lest one should wonder if the predicate hanasu 'speak' in generic interpretation might be taken as a type transfer from an SL predicate 'be speaking' and suggest that what we have observed about this IL predicate is due to this type transfer, let me give examples with predicative nominals, intrinsically IP predicates:

(31) (yo-no-naka mo kawatta mono da)

(world also changed thing is)

'(the fact is that the world has indeed changed)' 
Nihon-zin ga major league no sensyu de Mooko-zin ga sumoo tori da Japanese NOM GEN player and Mongolian NOM sumo wrestler is 'Japanese are major league players and Mongolians are sumo wrestlers' (yo no naka mo kawatta mono da) major no sensyu ga nihon-zin de sumoo tori ga mooko-zin da '(the fact is that the world has indeed changed) major league players are Japanese and sumo wrestlers are Mongolians'

The intended interpretation of (31) is not of course that all, or even many, major league players are Japanese, nor that all, or even most sumo wrestlers are Mongolian.

To recapitulate, whether we have a generic/universal construal or nongeneric/non-universal construal of a bare NP subject depends on context, that is, on domains of intended interpretation. Thus, what we have with IL sentences is a situation much like we have had above with SL sentences.9

Given these observations, I maintain that as in the case with SL sentences, whether a generic/universal or "non-generic/existential" interpretation is intended for the bare NP subject of an IL sentence is a matter of domains with respect to which the sentence is evaluated, and not the matter of whether the semantic representation of the sentence has a categorially distinct generic or existential NP as the subject. This point is obscured as long as our attention is turned only to generic sentences of the type which allow usual generic interpretation due to the way the real world actually is, such as (25)b above. (25)b can be interpreted as generic/universal whatever the domain of interpretation happens to be. Examples like (26) and (31) are crucial.

We might say that an IL sentence with a bare NP is construed as generic/universal if the intended domain of interpretation is a g-domain, and as nongeneric/non-universal (existential) if not. However, we cannot define g- and non-gdomains for IL sentences similarly to way we did for SL sentences. For one thing, it is not obvious that the introduction of an analogue of (19) for IL sentences, with a "state of affairs" argument in place of an event argument, is a viable solution. Furthermore, as is well known, a generic reading is in many ways different from a universal reading and defies any simplistic account in terms of set-theoretically defined extensions. Nor is it clear exactly how the non-generic/non-universal construal of a bare NP subject of an IL sentence should be understood. It is certainly an existential construal of some sort, but there is something generic about it, too; it might be called an existential generic. Mere existence does not suffice to license existential generics. For example, even though there are French speaking Americans, it would not be proper to say "Americans speak French" in the context of (25). I shall not attempt to interpret/define g-domains for IL sentences set/model theoretically. Instead, I adopt an axiomatic approach; by reversing the direction of conceptualization, I propose to understand g-domains for IL sentences as those that license generic/universal construals and non-g-domains as those that do not.

I maintain, then, on the strength of Assertions 1-4, which followed from (19), that the following analogues of Assertions 1-4 hold as axioms:

Analogue of Assertion 1. (Downward invariance of the g-domain) A subdomain of a g-domain for an IL proposition $\mathrm{p}$ with a bare NP subject is a $\mathrm{g}$ domain for p. 10

Analogue of Assertion 2. (Upward invariance of the non-g-domain) A domain that contains a non-g-domain for an IL proposition $\mathrm{p}$ with a bare NP subject is a non-g-domain for $\mathrm{p}$.

Analogue of Assertion 3. An IL sentence $S$ represents one and the same 
PROPOSITION through upward extension and downward restriction of domains.

Analogue of Assertion 4. A wa-marked IL sentence with a bare noun phrase subject is interpreted in a g-domain of the proposition represented by its source plain sentence.

These are plausible assertions, but since we lack grounding of the basic concepts on the standard set/model theoretic framework, they must be taken, and ultimately justified, as hypotheses for an empirical theory about g- and non-g-domains, or, equivalently, about generic/universal and non-generic/existential construal.

\section{The synthesis}

In the preceding two sections I have presented evidence for the claim that sentences with bare NP subjects, whether SL or IL, are subject to generic/universal or nongeneric/non-universal, existential construals depending on whether the domain of interpretation is a g-domain or not. I further maintain that parallel sets of assertions hold about these construals and domains for SL and IL sentences. A standard response to the situation we face might be to assume that both SL and IL sentences are semantically ambiguous, mapped onto two distinct semantic representations. Formally, such a standard semantic approach would adequately describe the observed distinctions. However, I would propose a different, pragmatic approach. I am not at present in a position to offer empirical evidence to choose one or the other approach on formal grounds. My argument is a plausibility argument speculating on performance.

Thus, as stated in Assertion 3 and its analogue above, I propose that a sentence with a bare NP subject represents one and the same proposition, but that its construal depends on where it is to be interpreted. I would attribute to the speaker and hearer more secure knowledge about sentences and propositions than merely how to interpret them, which depends on the choice of the domain for interpretation. Misunderstanding, if it takes place, is then due not to the failure of understanding one and the same sentence or one and the same proposition (meaning) represented by a sentence by the speaker and hearer, but to the failure to agree on the pragmatic choice of one and the same domain for the interpretation of the communicated proposition.

In view of the parallelism we observed above between SL and IL sentences with bare noun phrase subjects, I conclude:

Thesis 1. Sentences with bare NP subjects represent the same type of propositions, regardless of whether they are SL or IL.

Let us call bare NP subjects as well as sentences with bare NP subjects GENERIC/EXISTENTIAL. Generic/existential sentences satisfy the same set of axioms formulated in Assertions 1-4 and their Analogues whether they are SL or IL.

There is nonetheless a difference between the SL and the IL cases. For SL sentences, we can adequately account for both generic/universal and nongeneric/existential interpretation and for $\mathrm{g}$ - and non-g-domains in terms of the standard set/model theoretic approach to semantics. For IL sentences, we do not have a satisfactory account, either for generic/universal or for non-generic/existential interpretation; nor can we ground the concept of g-domain on a set-theoretic basis. This means that we cannot present a unified extensionalist account for the attempted generalization over the SL and IL distinction formulated in Thesis 1.

$\mathrm{Be}$ that as it may, I still have to formulate a generalization for the unified understanding of generic/universal and non-generic/existential interpretations for SL 
and IL sentences. Let us leave aside negative sentences, as we have in effect been doing in this presentation, since negation involves its own complications. Then, the valid generalization is: a sentence with a bare NP subject can be construed as representing a universal affirmative proposition in the sense of classical syllogism (that is, the $\mathrm{A}$ form in the traditional notation) when intended to be interpreted in a $\mathrm{g}$ domain, and a particular affirmative (that is, the $\mathrm{E}$ form) when intended to be interpreted in a non-g domain. A generic/existential proposition can be an input to an A-type inference if interpreted in a g-domain, and to an E-type inference when interpreted in a non-g-domain. Let me conclude:

Assertion 5. A generic/existential (affirmative) sentence is interpreted as the A form (universal) and the E form (particular) of a syllogism in a g-domain and non-gdomain, respectively, and respective syllogistic inference rules may apply.

Let us note that whatever the right LOGICAL representations of generic/universal and non-generic/existential interpretations might be for IL sentences, they cannot in general be taken as their semantic representations; the same point has been claimed for the logical representations of SL sentences in the form of existentially and universally quantified formulas of predicate calculus in section 3.2 above. Assertion 5 must be understood as entailing the following general claim:

Thesis 2. Logical representations of sentences are not semantic representations; they are pragmatically determined relative to domains of interpretation.

\section{Milsark's generalization(s)}

With the preceding preparations, we now proceed to discuss Milsark's generalization. I repeat it here in two forms, as before:

Milsark's generalization: Properties may only be predicated of "strong" NPs. (Milsark 1977:16).

Milsark's generalization (restricted to bare NPs and rephrased in terms of IL)

IL predicates may only be predicated of generic NPs.

Let us remind ourselves that Milsark's original definition of the "strong/weak" distinction is syntactic in character, in fact, dependent on English syntax: NPs are weak if they are permitted in post-copular position in there-insertion existential sentences; otherwise, they are strong (Milsark 1977: 8). The distinction, however, has been extended to other languages in later literature, on semantic grounds. For our present consideration of bare NPs, the distinction amounts to that between generic and existential. In any case, the original Milsark generalization concerns selectional restrictions that hold between predicate types and NP types. As far as bare NPs are concerned, I have maintained that the categorial selection between generic and existential is illusory, and hence so is that subpart of Milsark's generalization that relates to this distinction. I put this conclusion in the following thesis.

Thesis 3. Milsark's generalization (restricted form) is an epiphenomenon.

The argument for this thesis prima facie cannot be directly extended to cardinality NPs such as two apples, many apples etc. We need to clarify what we want to understand by "generic/universal cardinals." Be that as it may, I believe that Thesis 
3 can be generalized to the original form of Milsark's generalization in a full form, but in this paper, I put cardinality NPs aside and leave the matter only as a conjecture. 11

There is, however, a distinct difference between SL and IL sentences: SL sentences allow referential construal of their indefinite subject but not IL sentences.

Thesis 4. (The residue of Milsark's generalization) Indefinite NPs can be 'referential' in SL sentences but not in IL sentences.

Observe the contrast between (33) and (34) and between (35) and (36):

Look there, (many) apples are arranged beautifully in a basket!

*Look there, (many) apples are beautiful!

Look there, (many) Canadians are speaking French

*Look there, (many) Canadians speak French

Under Milsark's generalization, this restriction might be understood as a corollary: the referential reading is dependent on and derived from the existential reading. Milsark's generalization excluded existential readings from IL sentences, hence only SL sentences would be able to have referential readings.

This account, however, cannot be kept intact in our new perspective: we allow non-generic/existential readings for IL sentences as well, and hence the existential/non-existential distinction cannot be employed to single out SL sentences. Referentiability does not cut across the SL/IL distinction, that is, the event/property distinction. Referentialibility seems to be a fundamental characteristic feature of event-hood. The referential-nonreferential distinction amounts, it appears, to restating the definition of the IL/SL distinction; this is the significance of Thesis 4.

Let us try to derive this generalization directly from the SL/IL distinction. Let us recall the form of the representation of an SL proposition given in (19), which I repeat here as (37):

(37) Representation of an SL proposition p:

$\mathrm{NP}_{1} \ldots$... Pred...e...,

where $e$ is an event argument and NP is the subject of the predicate.

If we take e as a variable, and unselectively bind (37) we get an existential construal:

$$
\text { Existential reading: } \exists(\mathrm{e}, \mathrm{x})\left[\mathrm{NP}_{1}(\mathrm{x}) \ldots \text { Pred...e... }\right]
$$

If we take e as a constant and do not bind e, we assume that we get a referential construal:

Referential reading: $\left|\mathrm{NP}_{1}\right|_{\mathrm{s}, \mathrm{w}}=\left|\mathrm{NP}_{1}\right| \cap|\mathrm{e}|$, where $|\quad|$ indicates the "universal" valuation and ||$_{S, w}$ the interpretation relative to $S$ and a model/world w with respect to which the situation in question is described. $|e|$ denotes the set of entities involved in e.

The referential interpretation of $\mathrm{NP}_{1}$ falls out from the referential construal of the event argument e. We assume that this is the only way indefinite NPs get referential readings. That is, indefinite REFERENTIAL NPs are dependent on events.

So far, we have discussed Milsark's generalization within the bounds of the theory of propositions, where its original form belongs. Let us now move to the 
discussion of the perceived connection between Milsark's generalization and the theory of judgments. In Japanese, the subject of a categorical judgment (i.e., the waphrase of a $w a$-marked sentence) must be a semantically definite noun phrase, assuming that generic bare NPs are semantically definite. We seem to be able to state a generalization much like Milsark's: The subject of a categorical judgment must be strong.

But in our approach, this formulation is problematic; for, the strong/weak distinction as originally understood concerns a categorial distinction. But we do not distinguish generic (i.e., strong) bare NPs from existential (i.e., weak) bare NPs as a different category. We have only one kind of bare NPs; i.e., bare NPs are generic/existential NPs, though they may function pragmatically in distinct ways, as they could participate in the generic/universal type of inference or the nongeneric/existential type of inference, depending on pragmatic choices of domains. But according to Assertion 4 in section 3.4 and the Analog of Assertion 4 in section 4, a $w a$-marked bare NP, that is, a bare NP as the subject of a categorical judgment, must be interpreted in such a way that the wa-marked subject can be an input to a generic/universal inference. This condition must be characterized in terms of domains in our approach. To repeat, compare (40) and (41):

$$
\begin{aligned}
& \text { ringo wa kago no naka ni kirei ni narabete atta } \\
& \text { '(the) apples are arranged beautifully in a basket' } \\
& \text { *'there are apples arranged beautifully in a basket' } \\
& \text { ringo ga kago no naka ni kirei ni narabete atta } \\
& \text { '(the) apples are arranged beautifully in a basket' }
\end{aligned}
$$

The plain sentence (41) could be interpreted in a g-domain; in fact, I have even suggested that that is the default choice. Be that as it may, (41) can be interpreted in a non-g-domain as well. In contrast, the wa-marking of the subject in (40) excludes this possibility; the $w a$-marking signals that the interpretation is intended in a $\mathrm{g}$ domain. That means that the speaker must commit himself/herself to such a possibility of the subject term being an input to a generic/universal (A) type of inference. Likewise, compare (42) and (43):

$$
\begin{array}{ll}
\text { Canada-zin wa France-go o hanasu } & {[=(28)]} \\
\text { '(the) Canadians speak French' } & \\
\text { Canada-zin ga France-go o hanasu } & {[=(25) d]} \\
\text { 'Canadians speak French' } &
\end{array}
$$

The real world being what it is, the wa-marked sentence (42) can be accepted as a true statement only if the context is such that the generic statement "(the) Canadians present in the context speak French" is appropriate; that is, the context must be a gdomain. (43), on the other hand, can be taken as a true statement about the real world itself, which is not a g-domain. In contrast, (44) is an adequate statement in any context as long as the discourse concerns the real world:

$$
\begin{aligned}
& \text { France-zin wa France-go o hanasu } \\
& \text { 'The French speak French' }
\end{aligned}
$$

We thus have a Milsark type of constraint, restricted to bare NPs, transferred to the theory of judgment and formulated in pragmatic terms:

Thesis 5. (An analogue of Milsark's generalization) A categorical judgment must be interpreted in a g-domain of its subject. 
This thesis is related to the definiteness effect on categorical judgments mentioned earlier:

The definiteness effect on categorical judgments

(a) The subject of a CATEGORICAL JUDGMENT must be a "semantically definite" (including "generic") noun phrase.

(b) The subject of a CATEGORICAL JUDGMENT may not be (construed as) an indefinite "existential/cardinal" noun phrase.

"Definite" and "indefinite"are mutually exclusive and hence (b) follows from (a) as a corollary. The definiteness effect applies to non-bare definite noun phrases such as sono ringo 'that apple(s)' and proper names, but Thesis 5 appears not to, since $g$ domain has so far been defined only for bare noun phrases. But Thesis 5 can be understood to cover definite noun phrases as well by extending the concept of gdomain to definite noun phrases: indeed in conformity with the definition of definiteness, any domain where a definite noun phrase can properly be used may be understood as a g-domain for it. Then, Thesis 5 can be taken as equivalent to the formulation of the definiteness effect given in (a) above.

The advantage of Thesis 5 over the definiteness effect is that it does not refer to the definite/indefinite distinction, which is not syntactically marked in Japanese. We do not have to decide whether ringo 'apples' in (40), or, for that matter, Francezin in (44), either, is definite or not.

\section{Summary and conclusion}

In the view expressed here, sentences with bare NP subjects (or, equivalently, the propositions represented by them) are of one kind, generic/existential, representing the same type of propositions, whether they are SL or IL (Thesis 1); the different construals of the subjects, either as generic or existential, are a matter of pragmatic choices of domains. More generally, logical representations of sentences are not semantic representations (Thesis 2).

The original form of Milsark's generalization is about an epiphenomenon (Thesis 3) and needs no account (or, is accounted for by the recognition that it represents an epiphenomenon). However, we recognize a characteristic difference between SL and IL sentences with respect to the referential construal of indefinite NPs, the Residue of Milsark's generalization (Thesis 4). The original epiphenomenon is couched in the distinction between SL/IL predicates, but when this epiphenomenon disappears as an epiphenomenon, what is left, the residue, is what couched it.

Milsark's generalization originally belongs to the theory of propositions, but it seems to have commonly been perceived that it somehow relates to the theory of judgments; it might even have been expected that the theory of judgments accounts for Milsark's generalization. But Milsark's generalization, on closer examination, turns out to be an epiphenomenon; thus, nothing is left in the theory of propositions for which the theory of judgments could render service to it by deriving it. Nonetheless, we recognize a phenomenon that belongs to the theory of judgments which could be thought of as responsible for the perceived relation of Milsark's generalization to the theory of judgments. In our approach, this phenomenon can be identified as a matter of pragmatic choices of the domains for the interpretation of categorical judgments, An analogue of Milsark's generalization (Thesis 5). We have thus a generalization of a character quite different from Milsark's original, but I have retained Milsark's name for the sake of the perceived relation to its 
namesake. 12

\section{Endnotes}

* I would like to thank Chris Barker, Gregory Carlson, John Moore and Susan Fischer, who read early drafts of this paper and gave me valuable comments and advice.

1. At least for some predicates, the distinction between SL/IL must be understood relative to the sentences of which they are main predicates; in other words, the distinction is strictly speaking about sentences, and about predicates only relative to the sentences of which they are main predicates. In what follows I apply SL/IL to sentences and predicates interchangeably.

2. Cf: Kuroda (1965: 44). Ladusaw (1994) attempts to derive Milsark's generalization "from the assumptions involved in the ontology underlying the thetic categorical distinction." A step in his derivation corresponds to the definiteness effect cited here. Two formulations are given by Ladusaw:

(i) The subject of a categorical judgment cannot be a nonspecific indefinite; its reference is "presupposed." (First version).

(ii) The subject of the basis of a categorical judgment must be an object, not a description of an object. (Second Try)

(ii) may be compared with the following statement, where substance corresponds to Ladusaw's concept of object:

(iii) We might say that the 'topic' marker $w a$ functions to indicate that the entity referred to is cognitively apprehended as substance." (Kuroda 1992: 42)

3. See Kuroda (1965, Chapter 2; 1972; 1992, Chapter 1). The concepts CATEGORICAL JUDGMENT and THETIC JUDGMENT originate in the Brentano-Marty tradition of linguistic theory. They were applied to the account of the distinction between wamarked and plain sentences in Kuroda (1972), but this account, in terms of judgments, originates in Kuroda (1965; chapter 2), where the distinction is made between PREDICATIONAL JUDGMENT and NONPREDICATIONAL DESCRIPTION. A more detailed account of judgment and sentence forms is given in Kuroda (1992: chapter 1). Kuroda (2001) extends the concept of DESCRIPTION to IL sentences; the thetic judgment is a subtype of the description, with an SL predicate.

4. The definition $\left|N P_{1}\right|_{S, w}=\left|N P_{1}\right| \cap|e|$ given in (19) is generally adequate only for the case where Pred is a one-place predicate, as Chris Barker pointed out to me. Take, for example, $\mathrm{S}=$ 'fruits are beautifully arranged beside rotten apples'. Then, rotten apples being fruits, $\left|\mathrm{NP}_{1}\right|_{\mathrm{S}, \mathrm{w}}$ as defined in (19) would contain rotten apples as well. In order to avoid this difficulty, let $|e|_{\theta}$ be the set of entities that participate in $\mathrm{e}$ with the theta role $\theta$ of $\mathrm{NP}_{1}$ in $\mathrm{S}$, and let $\left|\mathrm{NP}_{1}\right|_{\mathrm{S}, \mathrm{w}}=\left|\mathrm{NP}_{1}\right| \cap|\mathrm{e}|_{\theta}$. According to this revision, no domain is a g-domain for $S=$ 'fruits are beautifully arranged beside rotten apples'; as desired. For our present purposes, we can restrict ourselves to oneplace predicates without losing generality.

5. This default case assumption is independent of the maximality condition associated with the exhaustive listing implicature. Assume that some apples and oranges are arranged in a basket. Given this context, (20) does not satisfy the maximality condition in this sense, and yet satisfies the default case assumption, if there is no apple that is not in the basket. Conversely, if there are some apples that are not in the basket, (20) does not conform to the assumption, and yet it could satisfy the maximality condition if apples but nothing else are in the basket. 
6. I argued that (i) cannot be assigned (ii), and for that matter any logical representation, at the semantic level:

(i)a ringo ga kago no naka ni kireini narabete atta apple NOM basket GEN in at beautifully arrange was

b 'apples were beautifully arranged in a basket'

$$
\exists x[\operatorname{APPLE}(\mathrm{x}) \& A R R A N G E D-I N-A-B A S K E T(x)]
$$

In contrast, one could argue that (iii) is effectively represented by (ii) at the semantic level, due to the special property of the predicate exist:

(iii)a. kago no naka ni kireini narabete aru ringo ga atta [=15] basket GEN in at beautifully arrange be apple NOM was/existed

b. 'apples that were beautifully arranged in a basket were/existed'

For, the universally quantified proposition that a g-domain licenses for (iii) would be:

$$
\forall \mathrm{x}[\operatorname{APPLE}(\mathrm{x}) \& \text { ARRANGED-IN-A-BASKET(x)つE(x)] }
$$

where $\mathrm{E}(\mathrm{x})$ is the existence predicate. Formula (iv) is a tautology and needs no representation for the semantic/pragmatic function of a sentence; (ii) suffices for (iii) as a logical representation in any domain.

Now, consider the English there insertion sentence (v):

(v) there are apples arranged beautifully in a basket

On the one hand, (v) is commonly used as a translation of the Japanese sentence (i)a as well as the logical formula (ii) into English. On the other hand, we observed above a functional parallelism between the inference (17)b in English using (v) and the inference (16) in Japanese using (iii)a. It is reasonable to assume, then, that (v) is a surface transform of (iii)b, and not of (i)b..

Thus, for there insertion sentences it is legitimate to assume that existentially quantified formulas like (ii) are assigned at the semantic level, even though they are not for plain SL sentences like (i)b.

7. Presupposing that it is a subdomain where $S$ is interpretable, that is, that it contains the event e.

8. Ringo wa in (23)a could refer to the apples that have already been mentioned in the course of the discourse, or the specific apples implicitly understood in the context, but it does not have to and in that case, the different effect of (23) attributable to $w a$ seems to be that somehow or other the presence of apples are expected at parties or a particular party of the topic in the discourse. According to native speaker consultants, under such circumstances, the use of the definite NP the apples is proper in English, even though it is not anaphoric.

9. Cohen (2001) also notes "generics [that] receive what appears to be an existential interpretation." His existential generics, however, are accompanied by emphasis or focus. (26), given in the context in (25), may also be taken as implicitly focused, but (31)-(32) are plain statements without focus and show that existential construals of IL sentences are not limited to those with emphasis or focus.

10. I am not certain what presupposition, if any, is required for a subdomain where IL proposition $\mathrm{p}$ remains interpretable; see note 7 for the condition that subdomains contain the event e for the SL case. This Analogue must be understood as an axiom 
in conformity with which the relevant sense of "subdomain" must be determined. 11. Besides, as far as Japanese is concerned, complications involved in the syntax and semantics of numeral or cardinality expressions must be worked out before any substantive arguments can be made on them. For example, the following two forms of slightly different syntax seem to show varied acceptability.

(Yononaka mo kawatta mono da)

('the world has changed)

(i) Nihon-zin ga san-nin (mo) yuumeina major no sensyu da. Japanese NOM three-CLS (also) famous major GEN player be '(As many as) three Japanese are famous major league players'

(ii) ?*San-nin (no) Nihonzin ga yuumeina major no sensyu da three-CLS GEN Japanese NOM FAMOUS major GEN player be 'three Japanese are famous major league players'.

12. The original talk I gave at the SALT meeting was entitled "Categorical and thetic judgments, Milsark's generalization and the definiteness effect." In a later part of the talk, I tried to elaborate on the contrast between the two types of "existential" sentences, illustrated by (12) and (15), respectively, and the implication of that contrast for how thetic judgments are expressed. I called a sentence of the latter type the existential transform of the corresponding one of the former type. Syntactic ecology, so to speak, of a particular language could influence the profiles of these truth-conditionally equivalent but logically not equi-functional sentence types in that language. I claim that in English existential transforms are represented by thereinsertion sentences at the surface level. See note 6 above. Now, the thetic judgment is often (but should not be exclusively) associated with "existential" sentences, and for English, in particular, with there-insertion sentences, therefore with existential transforms, in the sense introduced above. Due to this association, the thetic judgment appears also of ten associated with the "definiteness," or rather, indefiniteness effect. In contrast, in Japanese, the existential transform should count rather as a marked form, and as a consequence, the plain form is a normal expression for a thetic judgment; Japanese thus is clear of the perceived (in)definiteness effect of thetic judgments.

Paying attention to the functional contrast between sentences of the plain form and their existential transforms was instrumental for initiating the approach developed above. It would also be beneficial for a proper understanding of the thetic judgment beyond the bounds of truth conditional equivalence. But this topic is tangential to the main theme of this paper and I leave it for future work.

\section{References}

Carlson, Gregory N. 1977. Reference to Kinds in English. U. MA Dissertation. [Published from New York: Garland Press, 1980.]

Cohen, Ariel. 2001. Existential generics. Ben-Gurion University of the Negev, ms.

Kuroda, S.-Y. 1965. Generative Grammatical Studies in the Japanese Language, Dissertation, MIT. Facsimile edition, New York: Garland Press, 1979.

Kuroda, S.-Y. 1972. "The categorical and the thetic judgments; evidence from Japanese syntax," Foundations of Language 9.2:153-185.

Kuroda, S.-Y. 1992. Japanese Syntax and Semantics: Collected Papers. Dordrecht: Kluwer Academic. In particular, Chapter 1. Judgment forms and sentence forms.

Kuroda, S.-Y. 2001. Focusing on the matter of topic: a study of $w a$ and $g a$ in Japanese. Talk presented at Charles University, Prague. 
Ladusaw, William A 1994. "Thetic and categorical, stage and individual, weak and strong." In M. Harvey and L. Santelmann eds. Proceedings of SALT IV, Ithaca, NY, 220-229.

Milsark, G. L. 1977. Toward an explanation of certain peculiarities of the existential construction in English. Linguistic analysis 3.1-30. 